\title{
Hexa-X
}

\section{The European 6G flagship project}

Mikko A. Uusitalo

Nokia Bell Labs

Espoo, Finland

mikko.uusitalo@nokia-belllabs.com

Patrik Rugeland

Ericsson Research

Stockholm Sweden

patrik.rugeland@ericsson.com

Mauro Boldi

Telecom Italia

Turin, Italy

mauro.boldi@telecomitalia.it

\section{Emilio Calvanese Strinati \\ CEA Leti \\ Grenoble, France \\ Emilio.calvanese- \\ strinati@cea.fr \\ Gino Carrozzo \\ Nextworks \\ Pisa, Italy \\ g.carrozzo@nextworks.it \\ Panagiotis Demestichas \\ WINGS ICT Solutions \\ Athens, Greece \\ pdemest@wings-ict- \\ solutions.eu}

\author{
Mårten Ericson \\ Ericsson Research \\ Luleå, Sweden
}

marten.ericson@ericsson.com

Gerhard Fettweis

Technical University of Dresden

Dresden, Germany

Gerhard.Fettweis@tudresden.de

\section{Marie-Helene Hamon Orange \\ Rennes, France}

mhelene.hamon@orange.com

$$
\begin{gathered}
\text { Matti Latva-aho } \\
\text { University of Oulu } \\
\text { Oulu, Finland }
\end{gathered}
$$

matti.latva-aho@oulu.fi

Josep Martrat

Atos

Madrid, Spain

josep.martrat@atos.net

$$
\begin{gathered}
\text { Aarno Pärssinen } \\
\text { University of Oulu } \\
\text { Oulu, Finland } \\
\text { aarno.parssinen@ oulu.fi }
\end{gathered}
$$

\author{
Björn Richerzhagen \\ Siemens AG
}

Munich, Germany

bjoern.richerzhagen@siemens. elif.ustundag.soykan@ericsson.

com

Dario Sabella

Intel Deutschland GmbH

Munich, Germany

dario.sabella@intel.com

Hans D. Schotten

University of Kaiserslautern

Kaiserslautern, Germany

schotten@eit.uni-kl.de

Pablo Serrano

Universidad Carlos III de Madrid

Leganes, Spain

pablo@it.uc3m.es

Giovanni Stea

University of Pisa

Pisa, Italy

giovanni.stea@unipi.it

Tommy Svensson

Chalmers University of

Technology

Gothenburg, Sweden

tommy.svensson@chalmers.se
com
Elif Ustundag Soykan
Ericsson Research
Istanbul, Turkey

Gustav Wikström

Ericsson Research

Stockholm, Sweden

gustav.wikstrom@ericsson.com

$$
\begin{gathered}
\text { Volker Ziegler } \\
\text { Nokia Bell labs } \\
\text { Munich, Germany } \\
\text { volker.ziegler@ nokia-bell- } \\
\text { labs.com } \\
\text { Yaning Zou } \\
\text { Technical University of } \\
\text { Dresden } \\
\text { Dresden, Germany } \\
\text { Yaning.zou@ifn.et.tu- } \\
\text { dresden.de }
\end{gathered}
$$

\begin{abstract}
Hexa-X will pave the way to the next generation of wireless networks (Hexa) by explorative research $(X)$. The Hexa-X vision is to connect human, physical, and digital worlds with a fabric of sixth generation (6G) key enablers. The vision is driven by the ambition to contribute to objectives of growth, global sustainability, trustworthiness, and digital inclusion. Key 6G value indicators and use cases are defined against the background of technology push, society and industry pull as well as objectives of technology sovereignty. Key areas of research have been formulated accordingly to include connecting intelligence, network of networks, sustainability, global service coverage, extreme experience, and trustworthiness. Critical technology enablers for 6G are developed in the project including, sub- $\mathrm{THz}$ transceiver technologies, accurate standalone positioning and radio-based imaging, improved radio performance, artificial intelligence (AI) / machine learning (ML) inspired radio access network (RAN) technologies, future network architectures and special purpose solutions including future ultra-reliable low-latency communication (URLLC) schemes. Besides technology enablers, early trials will be carried out to help assess viability and performance aspects of the key technology enablers. The 6G Hexa-X project is integral part of European and global research effort to help define the best possible next generation of networks.
\end{abstract}

Keywords-6G, B5G, wireless, $6 G$ vision

\section{INTRODUCTION}

During 2030 and beyond, Europe and the world will face opportunities and challenges of growth and sustainability of tremendous magnitude; to pro-actively tackle issues of green deal efficiency, digital inclusion and assurance of health and safety in a post-pandemic world will be key. A powerful vision is needed to connect physical, digital, and human worlds, firmly anchored in future wireless technology and architectural research. The Hexa-X vision calls for an X-enabler fabric of connected intelligence, networks of networks, sustainability, global service coverage, extreme experience, and trustworthiness.

Wireless technologies are of critical relevance for our society and economy today; their importance for growth will continue to steadily increase with fifth generation (5G) and its evolution, enabling new ecosystems and services motivated by strongly growing traffic and trillions of devices [1-4]. The Hexa-X project ambition includes to develop key technology enablers in the areas of a) fundamentally new radio access 
technologies at high frequencies and high-resolution localization and sensing; b) connected intelligence though AIdriven air interface and governance for future networks, and c) 6G architectural enablers for network disaggregation and dynamic dependability.

Europe has been a leader in wireless network technologies for decades. It is now critical to initiate and consolidate our efforts in the joint research ambition of a "flagship" project to maintain and strengthen the global industry leadership for the $6 \mathrm{G}$ era. The Hexa-X flagship is a unique effort of vision, and an opportunity for disruptive impact in sustainable growth and technology experience leveraging the trustworthiness in Europe and worldwide.

This paper presents the European 6G flagship project Hexa-X that started beginning of January 2021 with a planned duration of 2.5 years. This is a first milestone in the development towards $6 \mathrm{G}$, see Figure 1.

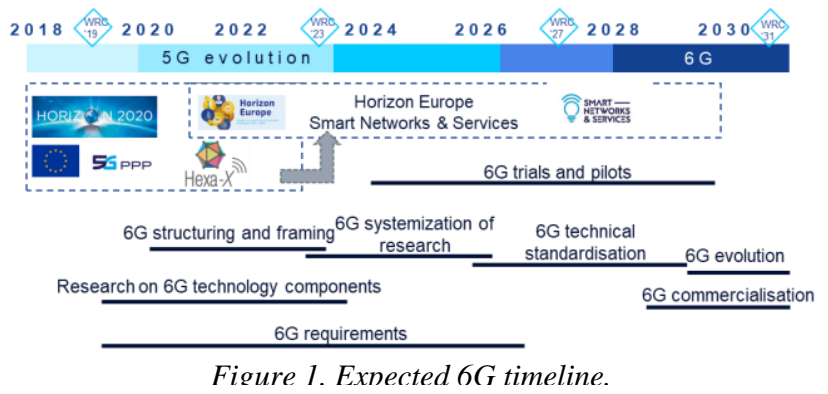

The project brings together key industry stakeholders and academia in Europe to take the lead in advancing 6G. The consortium includes top representatives of all stakeholders involved in this value chain, ranging from vendors, operators, IT industry, and high-tech companies. They together create a unique ecosystem capable to realize the Hexa-X main objectives and take on a global leadership role, see Figure 2.

Latest information is available from the project website https://hexa-X.eu/.

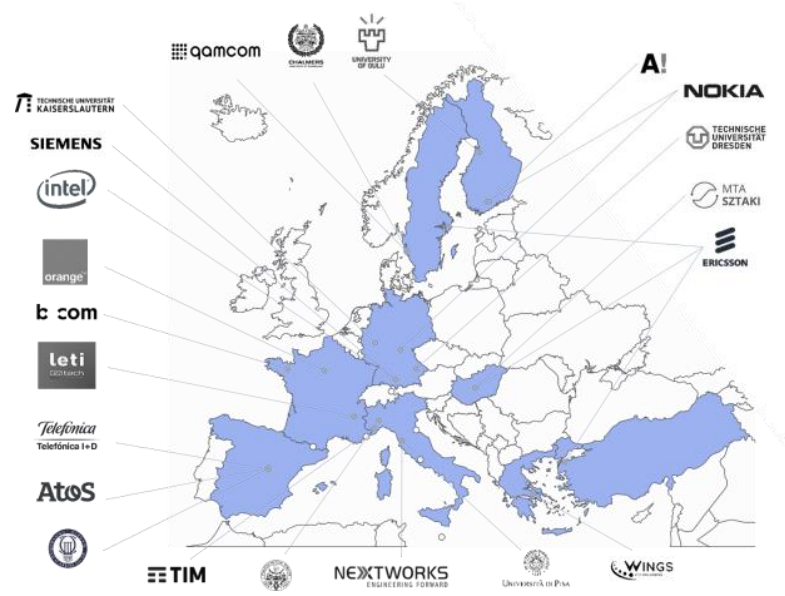

Figure 2. Hexa-X participants and countries in the consortium.

The rest of the paper is outlined as follows. Section II presents the vision of the project including key research challenges, which was jointly defined by the 25 participants in Hexa-X. Section III introduces the planned demonstrations that will showcase the tackling of research challenges, while
Section IV presents the project in the context of the external activities towards the $6 \mathrm{G}$ definition for establishing its role as a flagship project within the European $6 \mathrm{G}$ research and industry community.

\section{PROJECT VISION}

Hexa-X will define a vision for the $6 \mathrm{G}$ connectivity forming the backbone of a hyper-connected, data-driven society in beyond 2030 where sovereign citizens will be supported by a network of senses, autonomous things, and an increasingly intelligent environment. Protection of climate and environment, resource efficiency, enabling participation and inclusion, improving productivity, ensuring trustworthiness, and creating new business opportunities are key design criteria. To frame the challenges of research for communications in the 2030s, Hexa-X starts from the bold vision to connect and enhance interactions of the three worlds, Human, Physical, and Digital, as illustrated in Figure 3. The 2030s will offer tremendous opportunity and challenge to society and mankind worldwide. Contributing to objectives of global sustainability, trustworthiness and digital inclusion are a key part of the Hexa-X vision for the $6 \mathrm{G}$ era.

The Hexa-X key challenges aiming at both technology advancement and societal values of research have been formulated accordingly: Connecting intelligence, cognitive and physical augmentation with the human in control; Network of networks, integrating millions of sub-networks with resources such as communication, data- and AIprocessing, and localization and sensing optimizing the connectivity at different scales; Sustainability, addressing the critical challenges such as energy efficiency and $\mathrm{CO}_{2}$ footprint of network infrastructure [5] and to be complemented by innovation across industry sectors and use cases [6]; Global service coverage, where digital inclusion will be a key driver and enhanced by efficiency and affordability of solutions; Extreme experiences, pushing the boundaries of mobile connectivity in terms of bit rate, latency, capacity, and localization accuracy and co-design for sensing; Trustworthiness, building on novel technology buildingblocks and trust foundations to assure security, privacy, reliability, and resilience for the networks of the $6 \mathrm{G}$ era.

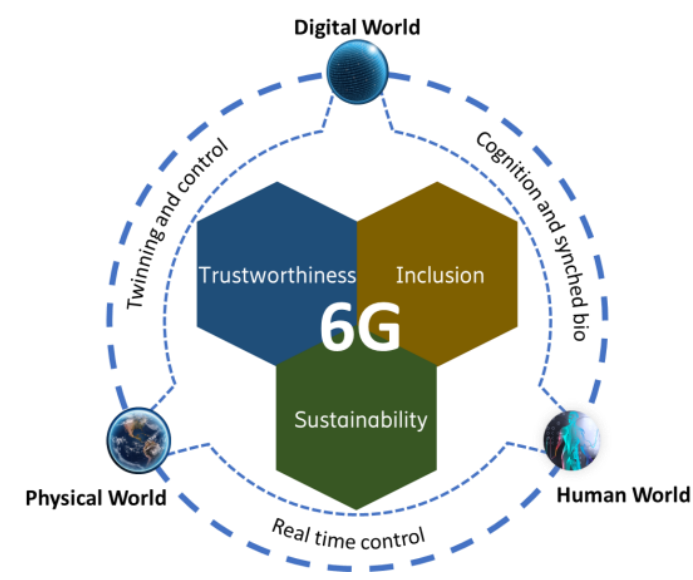

Figure 3. Hexa-X vision of $6 G$

To address the $6 \mathrm{G}$ research challenges, the Hexa-X project will 
design, develop, and evaluate a wide range of technological enablers, illustrated in Figure 4 a) Radio performance including advances in radio access technologies towards Tbps and high resolution localization and sensing by exploring sub$\mathrm{THz}$ frequencies [7]; b) Connecting intelligence by means of native integration of artificial intelligence for e.g. network orchestration and service management as well as dynamic datadriven air-interface design; and c) Network evolution and expansion to increase the flexibility and efficiency of networks, including specific verticals as well as facilitating the application of intelligent agents in the network by identifying and addressing roadblocks in the design.

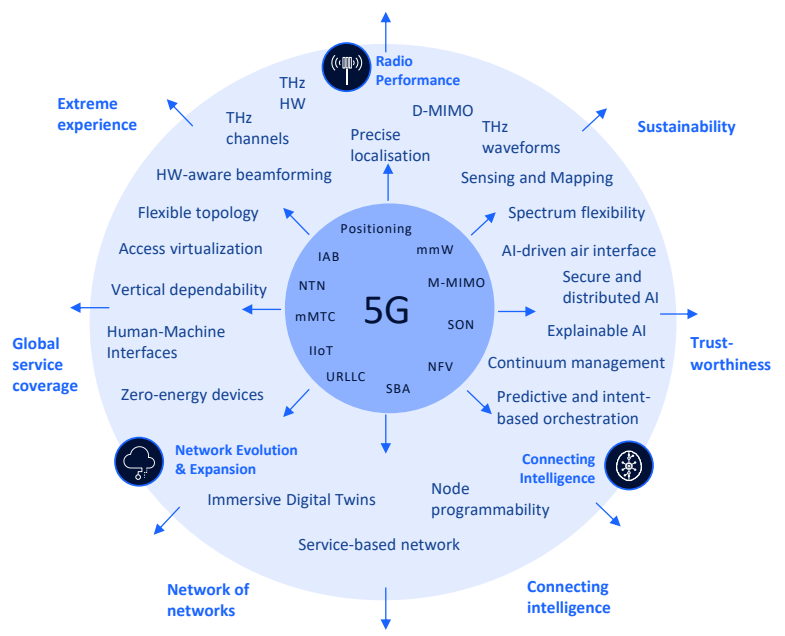

Figure 4. Hexa-X ambitions going from $5 G$ to $6 G$.

These technological enablers will be tied together in a framework - the $\mathbf{x}$-enabler fabric - which will be translated into a roadmap for future a $6 \mathrm{G}$ end-to-end system.

\section{A. Key use cases}

Hexa-X will identify use cases stemming from this vision and addressing the above six research challenges. In an initial step, five use case families have been identified, clustering various representative use cases as illustrated in Figure 5.

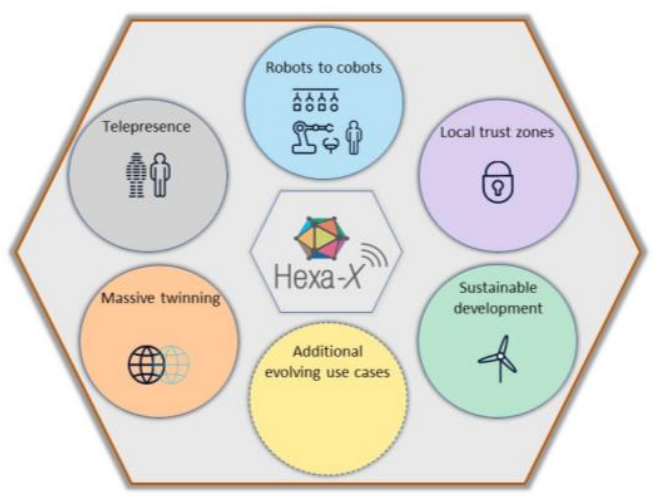

Figure 5. Hexa-X use cases families

As sustainability is a main driver in Hexa-X, this challenge is addressed by all families, and in particular in a dedicated one, Sustainable development. Trustworthiness is also an underlying challenge for all use case families, and more specifically for Local trust zones. Telepresence clusters use cases characterizing extreme experience, while Massive twinning and From robots to cobots involves different use cases building on the interconnection between the physical and digital worlds. This identification will continue during the lifetime of the project to refine and upgrade with relevant use cases.

\section{B. Key values and performance indicators}

In a $6 \mathrm{G}$ world, some of the existing key performance indicators (KPIs) will need to be extended to capture future needs, such as bit rate or connection density. But merely extrapolating the KPIs will not suffice to frame the $6 \mathrm{G}$ agenda of research, which needs to be considered end-to-end (E2E) to include some other aspects, such as energy efficiency or service availability. To scope 6G, key-value indicators (KVIs) will represent the dimensions of impact that go beyond the scope of deterministic performance measures into full account, such as sustainability, digital inclusion and trustworthiness. It is expected that $6 \mathrm{G}$ systems will incorporate novel aspects, such as integrated sensing, artificial intelligence, local compute-and-storage, and embedded devices. These aspects will both facilitate enhancements to existing KPIs, as well as require a range of new KPIs and KVIs which have not traditionally been associated with mobile networks, such as sensing accuracy, computational round-trip-time, AI model convergence time, etc. Moreover, as a foundational objective for all future technology, the United Nations Sustainable Development Goals (SDGs) will be important for Hexa-X and as an inspiration as well as a measuring stick. The Hexa-X project foresee a substantial enabling impact on most of the SDGs. Pull from new use cases and push from novel technologies will also impact a set of expanded and new set of capabilities in 6G, as illustrated in Figure 6.

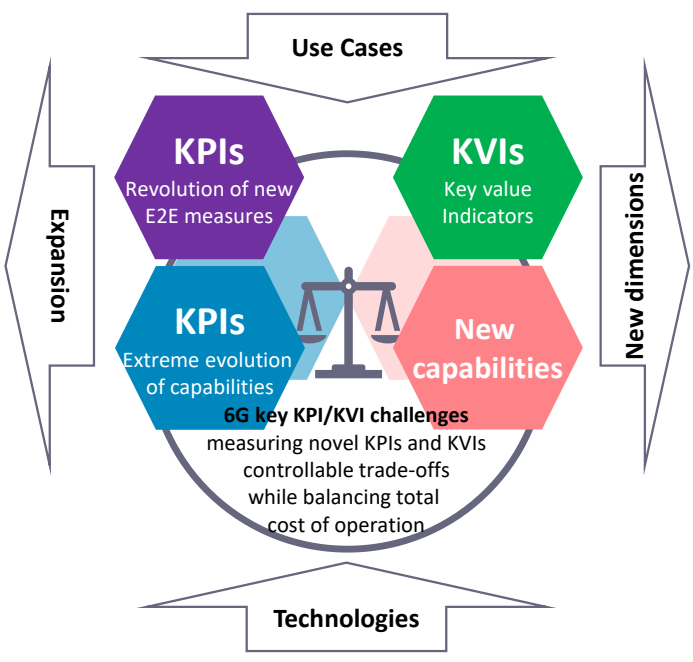

Figure 6. Key value and performance indicators.

\section{DEMONSTRATING 6G}

The Hexa-X project will showcase the technological enablers and the progress towards accomplishing the $6 \mathrm{G}$ vision by setting up five separate demonstrations (demos). 
Demo \#1: 6G OTA - Waveforms in action: The demo verifies selected $6 \mathrm{G}$ candidate waveforms designed in Hexa-X and evaluates their performance over the air (OTA) in realworld environment with non-ideal radio frequency (RF) and radio channel as seen in Figure 7.

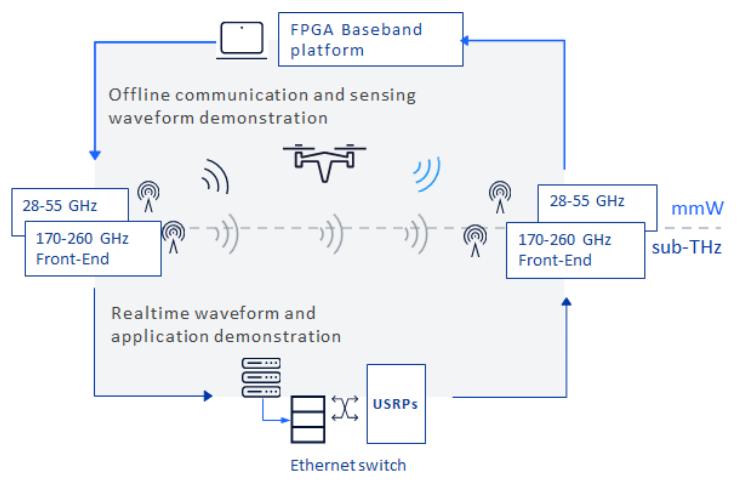

Figure 7. 6G OTA - Waveforms in action, demo \#1.

The work will be carried out by connecting three testbeds provided by Qamcom, University of Oulu, and TU Dresden within the project in three progressive phases using 6G waveform candidates: a) Non-real time demonstration of radar sensing performance at $\mathrm{mmW}$; b) Non-real time demonstration of communications and sensing performance at $200 \mathrm{GHz}$ region; c) Real-time demonstration of communications and sensing performance. The testbeds verify and evaluate the candidate waveforms in realistic environment to help select the best candidates for future studies. As waveforms strongly impact also RF transceiver performance and power consumption the results will be applied to radio system design for communications and sensing in all aspects studied in the Hexa-X project.

\section{Demo \#2: FED-XAI - Federated XAI demo:}

Hexa-X will demonstrate the developed framework for the federated learning of eXplainable AI (FED-XAI) models [8], along with the related signalling, using a real-time network emulator and real terminals as seen in Figure 8.

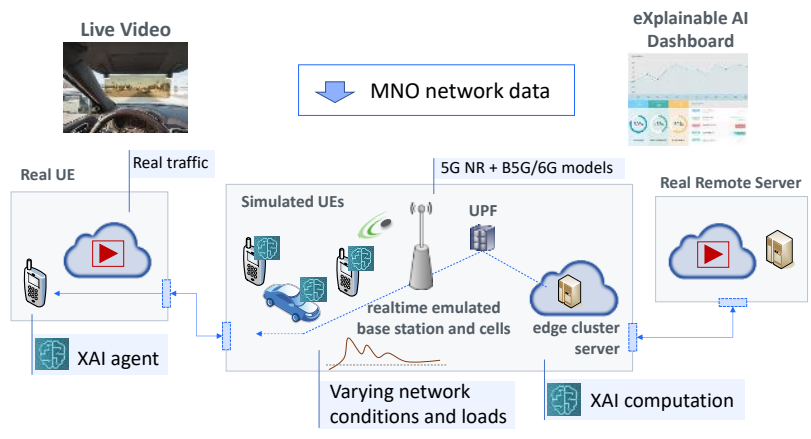

Figure 8. FED-XAI - Federated eXplainable AI demo, demo \#2.

End-user agents will sense local data and collaboratively train an XAI model with the goal to make predictions, e.g., on Quality of Service (QoS), without exposing their data to other agents. The model obtained from the federated learning process will be transferred to other agents, which will adapt it locally to perform predictions. Moreover, an edge side-agent, federated with the end-user ones, and possibly with agents from other
Mobile Network Operators (MNOs), will display in real time a dashboard, showing the predicted QoS in the federated domain and explaining how these have been achieved.

Demo \#3: Flexible topologies (FLEX-TOP) for efficient network expansion and complementary means for global coverage, sustainability and trust:

The demo will deliver insights on the efficiency of the flexible topologies concept as seen in Figure 9.

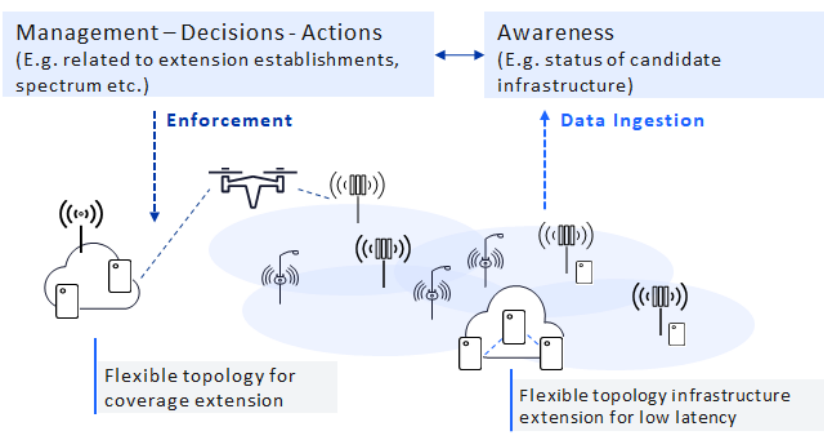

Figure 9. Flexible topologies (FLEX-TOP), demo \#3.

The key benefits of a flexible topology will be coverage extensions; service provision with lower latencies, since local structure is terminated at the infrastructure edge; security as the engagement is limited to selected devices; and lower energy consumption at the infrastructure. The management of the flexible topology will leverage on mesh/ad hoc/device-todevice (D2D) networking, disaggregated devices with the ability to flexibly allocate functionality or computing resources, as well as usage of ultra-high spectrum also in coordination with the infrastructure.

Demo \#4: Extreme performance in handling unexpected situations in industrial contexts:

The demo will target the handling of unexpected situations in industrial environments through $6 \mathrm{G}$ enablers as seen in Figure 10, involving infrastructure and mechanisms for emulating impairments.

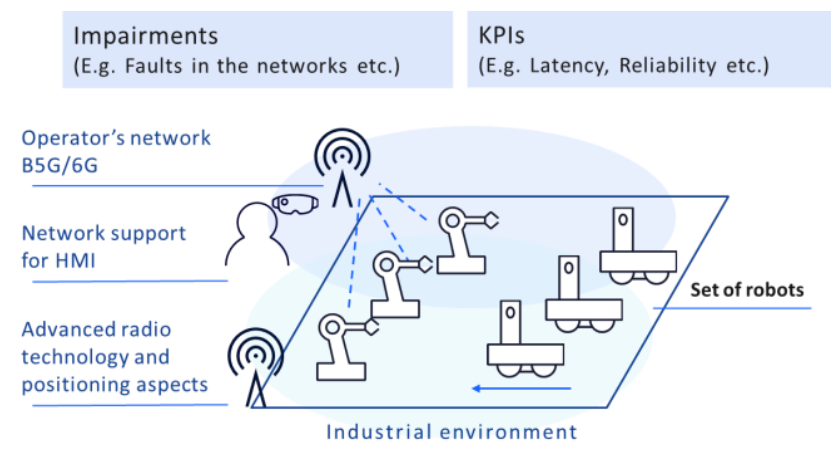

Figure 10. Extreme performance in handling unexpected situations in industrial contexts, demo \#4.

The infrastructure will be a set of robots (e.g., swarms), cooperating on a task, and $6 \mathrm{G}$ connectivity. Impairments will be faults in the network, robotic devices, or functionality. Several cases will be shown: a) using flexible topology, advanced network infrastructures, and predictive orchestration for mitigating impact of a faulty device by redistributing 
functionality and roles; b) using $6 \mathrm{G}$ to deliver high rate, low latency, and reliability supporting human-machine interfaces (HMI) [9]; c) using advanced radio and positioning to address impairments.

Demo \#5: Algorithms for data-driven device-edge-cloud continuum management:

A set of algorithms are required to support zero perceived latency in $6 \mathrm{G}$ networks. This demonstration will showcase the prediction of a future state of a device and necessary actions that will be taken for the service self-adaptation, to ensure lowest possible latency, reliability, and efficiency (in terms of resources, means used). In this regard, the algorithms will predict the next device state, based on its context (see Figure 11), e.g., user profile, network hints, application and IoT aspects. The actions will relocate the service as close as possible to the device.

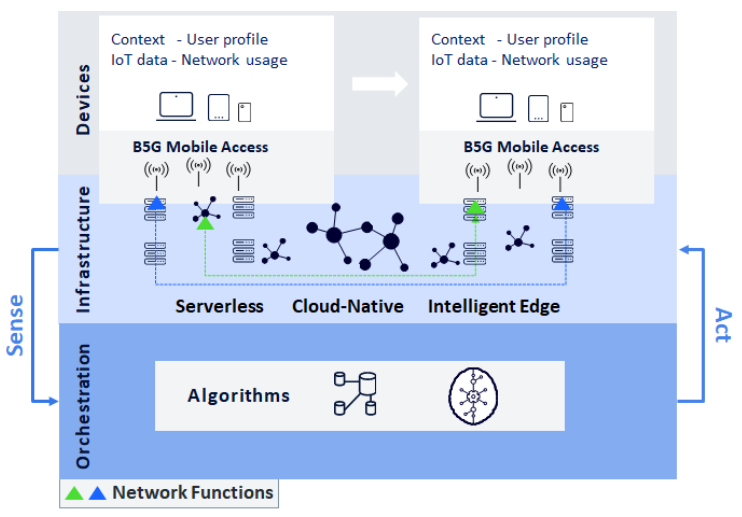

Figure 11. Algorithms for data-driven device-edge-cloud continuum management. demo \#5.

\section{OUTLOOK}

As the flagship project of the European $6 \mathrm{G}$ research and industry community, Hexa-X will actively exchange and align its vision with the concurrent research activities in the European and international context (see Figure 12). Building on the novel $\mathrm{x}$-enabler fabric, Hexa-X will be able to learn from other $6 \mathrm{G}$ - related research projects and integrate their inputs together with Hexa-X research outputs for achieving maximised impact among the research community, regulatory and standardisation fora.

Hexa-X will collaborate with a wide range of stakeholders at the European and international levels: from national R\&I Action Plan for 6G in Europe (e.g., 6G Flagship initiative in Finland), national $6 \mathrm{G}$ programs outside Europe (e.g. in the US, China, South Korea and Japan), policy makers (e.g., the European Commission), standard development organisations, (e.g., 3GPP, ITU and ETSI), to Hexa-X Advisory Group with top experts from society and sociology (UN, Uni Luxembourg), business verticals (ABB, BMW, SNCF, Thales) as well as other major 5G/6G industry representatives (CMRI, NTT DOCOMO, KT, Vodafone, Sony, Marvell, Qualcomm, Facebook, and CeWIT India). The forthcoming Smart Networks \& Services Joint Undertaking in the framework of the $5 \mathrm{G}$ Industry Association is a natural environment where the activities of Hexa-X will be fundamental for the success of the European 6G partnership [10].

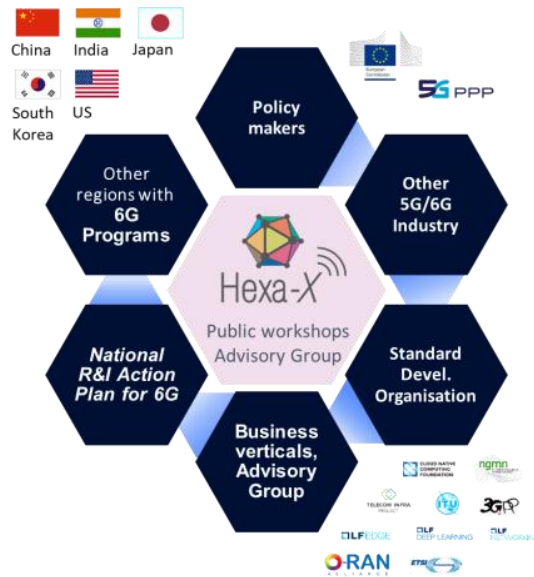

Figure 12. Relation of Hexa-X to major stakeholders.

\section{ACKNOWLEDGMENT}

Part of this work has been funded from the European Union's Horizon 2020 research and innovation programme under grant agreement No 101015956 Hexa-X. The authors would like to acknowledge the contributions of their colleagues in Hexa-X.

\section{REFERENCES}

[1] H. Viswanathan and P. Mogensen, "Communication in the 6G Era," IEEE Access, Bd. 8, pp. 57063-57074, 2020.

[2] G. Wikström et al. "Ever-present intelligent communication, A research outlook towards 6G", Ericsson, November 2020 [Online]. Available: https://www.ericsson.com/en/reports-and-papers/white-papers/aresearch-outlook-towards-6g.

[3] S. M. Atif et al. "Broadband Infrastructure and Economic Growth: A Panel Data Analysis of OECD Countries", ZBW, 2012.

[4] M. Latva-Aho, M.; Leppanen, K. (Eds.), "Key Drivers and Research Challenges for 6G Ubiquitous Wireless Intelligence", 2019.

[5] United Nations, The Sustainable Development Goals Report, 2019, [Online]. Available: https://unstats.un.org/sdgs/report/2019/TheSustainable-Development-Goals-Report-2019.pdf

[6] P. Bergmark, J. Malmodin. "Exploring the effect of ICT solutions on GHG emissions in 2030", [Online]. Available: https://doi.org/10.2991/ict4s-env-15.2015.5, 2015.

[7] T. S. Rappaport, Y Xing, O Kanhere, S Ju, A Madanayake, S Mandal, "Wireless communications and applications above $100 \mathrm{GHz}$ : Opportunities and challenges for 6G and beyond", IEEE Access 7, pp. 78729-78757, 2019.

[8] A. Fernandez, F. Herrera, O. Cordon, M. J. del Jesus, and F. Marcelloni, "Evolutionary fuzzy systems for explainable artificial intelligence: Why, when, what for, and where to?". IEEE Computational Intelligence Magazine, 14(1), 69-81, 2019.

[9] X. Gu et al., "EEG-based Brain-Computer Interfaces (BCIs): A Survey of Recent Studies on Signal Sensing Technologies and Computational Intelligence Approaches and Their Applications," in IEEE/ACM Transactions on Computational Biology and Bioinformatics, doi: 10.1109/TCBB.2021.3052811.

[10] W. Mohr. "Smart networks and Services task force - Introductory Statements, Smart Networks and Services Partnership Stakeholder workshop", 2019. 